\title{
Treatment of liver cancer in mice by the intratumoral injection of an octreotide-based temperature-sensitive gel
}

\author{
LILI ZHANG $^{1}$, SU YU ${ }^{1}$, ZHIJUN DUAN ${ }^{1}$, QIUMING WANG ${ }^{1}$, GE TIAN $^{2}$, YAN TIAN $^{2}$, WEI ZHAO $^{3}$, \\ HUI WANG ${ }^{4}$, CUILING ZHANG ${ }^{5}$, SHIBIN GUO ${ }^{6}$, QIGUI LIU $^{7}$, GAOHONG HE $^{8}$, TENGFEI BIAN ${ }^{8}$, \\ JIUYANG CHANG ${ }^{9}, \mathrm{XUE} \mathrm{JIN}^{1}$ and DONGSHENG CUI ${ }^{1}$
}

\author{
${ }^{1}$ Department of Gastroenterology, The First Affiliated Hospital, Dalian Medical University; ${ }^{2}$ Department of Clinical Pharmacy, \\ College of Pharmacy, Dalian Medical University; ${ }^{3}$ Morphology Laboratory, College of Basic of Medical Sciences, \\ Dalian Medical University; Departments of ${ }^{4}$ Ultrasound, ${ }^{5}$ Clinical Laboratory and ${ }^{6}$ Digestive Endoscopy, \\ The First Affiliated Hospital, Dalian Medical University; ${ }^{7}$ Dalian Medical University School of \\ Public Health Research Statistics, Dalian, Liaoning 116011; ${ }^{8}$ Institute of Chemical Engineering, \\ Dalian University of Technology, Dalian, Liaoning 116024; ${ }^{9}$ Department of Anesthesiology, \\ Dalian Medical University, Dalian, Liaoning 116011, P.R. China
}

Received May 15, 2013; Accepted August 21, 2013

DOI: $10.3892 /$ ijmm.2013.1542

\begin{abstract}
Octreotide (OCT) can inhibit tumor growth with few side-effects. In this study, we hypothesized that an OCTand poloxamer 407 (P407)-based temperature-sensitive gel may compensate for the short half-life of OCT, which may thus lead to the development of a novel therapy for patients with end-stage liver cancer by intratumoral injection. The proliferation and apoptosis of mouse Hca-F hepatocellular carcinoma cells were determined by MTT assay and Annexin V-PI staining. A mouse model of hepatocellular carcinoma was established by the subcutaneous transplantion of Hca-F cells and OCT-P407 or OCT solution were injected into the tumors, followed by the detection of OCT levels by high performance liquid chromatography (HPLC) over a specific time period. OCT-P407, ethanol, OCT, P407 or normal saline (NS) were injected into the tumors and the tumor size, weight and inhibition rate were measured 8 days later. Additionally, the expression of somatostatin receptor-2 (SSTR-2), vascular endothelial growth factor (VEGF) and caspase-3 was detected by immunohistochemistry and RT-PCR. Compared with the OCT group, the tumor inhibition rate and the apoptotic rate in the OCT-P407 group were higher and the effects were longer. The tumor size and weight
\end{abstract}

Correspondence to: Professor Zhijun Duan, Department of Gastroenterology, The First Affiliated Hospital, Dalian Medical University, No. 222 Zhongshan Road, Dalian, Liaoning 116011, P.R. China

E-mail: cathydoctor@sina.com

Key words: octreotide, poloxamer 407, Hca-F cells, intratumoral injection in the OCT-P407 group were lower and the tumor inhibition rate higher compared with the OCT, P407 and NS groups, with the exception of the ethanol group. The protein and mRNA expression of SSTR-2 and caspase-3 in the OCT-P407 group was higher, and that of VEFG was lower compared with the other groups, with the exception of the ethanol group. In the present study, we demonstrate that the intratumoral injection of OCT-P407 maintains OCT local effective concentration and prolongs its action time, with a greater therapeutic effect than that of OCT on its own. Although ethanol is more effective in certain aspects, its tumor inhibitory effects are similar to OCT-P407 and as such, OCT-P407 may be a suitable alternative.

\section{Introduction}

Hepatocellular carcinoma is a serious disease which develops rapidly and is difficult to detect at an early stage. Only $10-30 \%$ of patients can be treated by surgery $(1,2)$ and for those who cannot, targeted therapy is considered a good choice which has always been a hot spot of research.

Transcatheter arterial chemoembolization (TACE), percutaneous ethanol injection (PEI) and radiofrequency ablation (RFA) play important roles in the treatment of both early and advanced stage hepatocellular carcinoma. However, since many advanced stage patients suffer from hepatic dysfunction and such therapies may worsen the hepatic function, leading inclusively to hepatic failure, such applications are avoided. Among these techniques, PEI, a method of percutaneous intratumor injection, is used to treat the tumor mainly by the injection of ethanol, which causes protein denaturation, dehydration of the cancer cells and coagulation of small vessels within the tumor. Nevertheless, some patients are allergic to ethanol and the osmotic ability of ethanol has been found to be limited; hence, larger doses and repeated multiple-site injections are required in order to achieve complete tumor necrosis, 
which inevitably increases the likelihood of a variety of side-effects (3-5). On the other hand, the intratumoral injection of chemotherapeutic drugs for the treatment of hepatocellular carcinoma has been extensively studied (6); however, the drugs injected into the tumor do not diffuse evenly. Studies have shown that the diffusion is even and the release is delayed when poloxamer 407 (P407)-based thermosensitive gels are added to the injected drugs $(7,8)$. In recent years, the anticancer effects of somatostatin (SST) and somatostatin analog (SSTA) have gained increasing attention, with studies showing that the SSTA, octreotide (OCT), can significantly inhibit the growth of tumor cells and shrink the size of the tumor with prolonged intravenous application (9-11). Compared with SST, OCT has a longer half-life, stronger effects and fewer adverse effects. Targeted intratumoral injection therapy can further improve the therapeutic efficacy (12); however, for use in the treatment of advanced hepatocellular carcinoma by intratumoral injection, the half-life of OCT is still too short and frequent applications will add to the economic, physical and mental burden of patients.

In the present study, we hypothesized that P407 as a carrier may be combined with OCT to obtain OCT-P407 as a sustained-release preparation, which can make up for the shortcomings of OCT. At the same time, we established a mouse model of hepatocellular carcinoma by the subcutaneous transplantion of Hca-F cells to confirm the effects of this new agent (administered by intratumoral injection) on hepatocellular carcinoma. The findings of the present study may provide patients with advanced hepatocellular carcinoma with a novel, well-tolerated treatment method administered by intratumoral injection.

\section{Materials and methods}

Animal care. Kunming mice (weighing 18-22 g) were obtained from the Experimental Animal Center of Dalian Medical University, Dalian, China. The present study was carried out in strict accordance with the recommendations in the Guide for the Care and Use of Laboratory Animals of the Canadian Council on Animal Care. The protocol was approved by the Committee on the Ethics of Animal Experiments of Dalian Medical University (permit no. SCXK 2008-0002). All surgical procedures were performed under ether anesthesia and all of the subcutaneous inoculations and tumor injections were administered in a quiet and comfortable environment.

Animal model. The mice were housed in a specific pathogenfree (SPF) environment maintained at $24-26^{\circ} \mathrm{C}$ with a relative humidity of $60-65 \%$. They were allowed free access to water and food throughout the experimental period.

During the study, we tried our best to reduce the pain and suffering of the mice. The animals ate and drank freely in a comfortable and quiet environment while the mouse models of liver cancer were established by the subcutaneous transplantion of cancer cells. After the injection of the solutions to the 4 groups for 8 days, the mice were sacrificed. All the experiments were performed under diethyl ether anesthesia and the in vivo experiments conformed to the Medical Laboratory Animal Guidelines. This study was performed in compliance with the Helsinki Declaration. The surgical procedures were approved by the Animal Care and Use Committee of Dalian Medical University.

\section{Materials}

Cell line. The mouse hepatocellular carcinoma ascites cell line with a high lymphatic metastatic potential, Hca-F, was established and preserved in the Morphology Laboratory of Dalian Medical University.

Experimental animals. The SPF mice used in this study were provided by the Experimental Animal Center of Dalian Medical University.

Reagents. OCT powder and OCT standard preparation (Xinlibang Bio-Pharmaceutical Co., Chengdu, China; purity $>99 \%$ ); P407 and OCT-P407 were prepared by the College of Pharmacy of Dalian Medical University and the Dalian University of Technology, at a concentration of $0.1 \mathrm{mg} / \mathrm{ml}$; RPMI-1640 culture medium (HyClone Co., Logan, UT, USA); methyl thiazolyl tetrazolium (MTT) (Amresco LLC, Solon, OH, USA); Annexin V-FITC kit (Jingmei BioTech Co., Shenzhen, China); RT-PCR amplification kit (Thermo Fisher Scientific Inc., Waltham, MA, USA); rabbit anti-mouse VEGF antibody was acquired from Thermo Fisher Scientific Inc.; rabbit anti-mouse caspase-3 antibody (Boster Biotechnology Inc., Wuhan, China); the ready-to-use Immunohistochemistry kit and DAB coloration kit were acquired from Beijing Zhongshan Golden Bridge Biotechnology Co. (Beijing, China).

\section{Methods}

Preparation of thermosensitive gel and OCT-P407. The P407based thermosensitive gel consisted of P407, cellogran and other excipients, such as PEG-PLA block copolymer, ethylhydroxyethylcellulose and PEG-polymer-PLC block copolymer. OCT solution of the required concentration was added to the P407-based thermosensitive gel and incubated overnight at $4^{\circ} \mathrm{C}$ after gentle stirring. A colorless and transparent viscous liquid (OCT-P407) was thereby formed. OCT-P407 was placed in an ice water bath before use.

\section{In vitro experiments}

Cell culture. Hca-F cell counting, passage, culture, recovery and cryopreservation were performed as previously described $(13,14)$. Cells expanded as a single-layer suspension when observed under an inverted phase-contrast microscope (Olympus Co., Tokyo, Japan).

Measurement of cell proliferation. Screening for the optimum concentration of cells was performed as previously described (15). Groups were defined as follows $(n=5)$ : the control group used for colorimetric zeroing, where no cells were inoculated and only solution was added; the negative control group, where the cells were inoculated, but no solution was added; the OCT-P407 group, which was divided into 4 subgroups according to the different OCT concentrations $(0.1,1,10$ and $100 \mu \mathrm{g} / \mathrm{ml})$; the OCT group, defined as the positive control group, divided into 4 subgroups containing the same OCT concentrations as in the OCT-P407 group; the P407 group, which was the same as the OCT-P407 group except that OCT-P407 was replaced by P407. There were 4 parallel wells 
corresponding to each OCT concentration. The calculation of the inhibition rate (IR) was carried out according to the formula: IR = (1 - OD value of the experimental well/OD value of the control well) $\times 100 \%$. The $50 \%$ inhibitory concentration (IC50) was calculated by the modified Karber method according to the formula: $\operatorname{LgIC} 50=\mathrm{Xm}-\mathrm{I}[\mathrm{P}-(3-\mathrm{Pm}-\mathrm{Pn}) / 4]$, where $\mathrm{Xm}$ represents the $1 \mathrm{~g}$ of the maximum dose; I represents $1 \mathrm{~g}$ (maximum dose/adjacent dose); P represents the sum of positive response rates; $\mathrm{Pm}$ represents the maximum positive response rate; and Pn represents the minimum positive response rate. After the optimum concentration of cells and optimum inhibitory concentration were calculated, cell suspension was inoculated into a 96-well plate. OCT solution, OCT-P407 and P407 containing the same concentrations of OCT $(20 \mu \mathrm{g} / \mathrm{ml})$ were added separately. Three 96-well plates were placed in a $37^{\circ} \mathrm{C}$ incubator with $5 \% \mathrm{CO}_{2}$ and saturated humidity and taken out separately after 12,24 and $48 \mathrm{~h}$. After the addition of $20 \mu \mathrm{l}$ of $500 \mu \mathrm{g} / \mathrm{ml}$ MTT into each well, the cells were cultured for an additional $4 \mathrm{~h}$. The cells were then centrifuged at $1,000 \mathrm{rpm}$ for $10 \mathrm{~min}$, the supernatant was aspirated carefully and $150 \mu \mathrm{l}$ of dimethyl sulfoxide (DMSO) were added to each well. The plates were then shaken on a micro oscillator for $15 \mathrm{~min}$, after which the absorbance (OD) was detected using a microplate reader at a wavelength of $492 \mathrm{~nm}$. The OD detection was in duplicate and the values averaged. The growth inhibition ratio of each group was calculated by the IR formula.

Evaluation of apoptosis. Hca-F cells in the exponential phase were collected and the cell concentration adjusted to $1 \times 10^{6}$ cells $/ \mathrm{ml}$. The cells were divided into 4 groups as follows: negative control group, only cells were inoculated and no solutions was added; the OCT-P407, OCT and P407 groups, where the concentration of the solution was the optimum inhibitory concentration detected by MTT assay. After the cells were cultured with the different solutions for 12, 24 and $48 \mathrm{~h}$, apoptosis was detected by Annexin V-FITC/PI staining and flow cytometry (on a Becton-Dickinson cytometer) according to the manufacturer's instructions as previously described (16). Changes in cell morphology were detected and photographed under an inverted optical microscope after $48 \mathrm{~h}$ of culture.

\section{In vivo experiments}

Establishment of a mouse model of liver cancer by subcutaneous transplantion of cancer cells. Second generation of ascites liver cancer cells (with a survival rate $>98 \%$ ) was collected, washed twice with phosphate-buffered saline (PBS; $0.2 \mathrm{ml})$ and the cells were adjusted to $2.0 \times 10^{6} / \mathrm{ml}(17,18)$. One hundred mice were subcutaneously injected with $0.2 \mathrm{ml}$ of cell suspension $\left(\sim 5 \times 10^{6}\right.$ cells $)$ in the left axillary fossa and the tumor growth was monitored. Seven days after the injection, tumor diameter was measured with a slide caliper rule. Mice whose tumor diameter was $2 \mathrm{~cm}$, were used for the detection of the $\mathrm{P} 407$ retention time in the tumor cavity, whereas 50 mice whose tumor diameter was $1 \mathrm{~cm}$ were used for the detection of the therapeutic effects of the intratumoral injection of the different solutions.

Intratumoral injection. Fur around the tumor was shaved and the mice were anaesthetized with ether and placed in a supine position. The SP6-12 probe of Siemens Color Doppler ultra- sound system (116834 type; Siemens Co., Munich, Germany) was used to scan the position of the tumor. Following routine sterilization and draping, the solutions were injected with a needle of a $1 \mathrm{ml}$ syringe into the center of the tumor under ultrasound guidance. The needle was appropriately adjusted during the injection and the solution was slowly injected from deep to shallow, in order to distribute it homogeneously in the tumor cavity and the position of the needle was appropriately adjusted during the injection. The whole process was monitored in real-time and recorded by ultrasound.

Detection of $P 407$ retention time in the mouse tumor cavity. Methylene blue solution was mixed with P407 and normal saline solution (NS) separately to obtain methylene blue-P407 and methylene blue solution. Sixteen mice whose tumor diameter was $2 \mathrm{~cm}$ were randomly divided into the methylene blue-P407 and methylene blue solution groups, each group containing 8 mice. Under ultrasound guidance, the 2 reagents mentioned above were slowly injected into the center of the tumor and the pigmentation status of the skin around the tumor was observed. Twenty-four hours after the injection, the mice were culled and the tumors excised. The pigmentation status of the solid tumors inoculated with the 2 reagents was compared. Forty-eight mice whose tumor diameter was $2 \mathrm{~cm}$ were selected for receiving intratumoral injections under ultrasound guidance. Mice were randomly divided into the OCT-P407 and OCT groups, according to the solutions that were injected $(n=24)$ and at 12,24 and $48 \mathrm{~h}$ after injection, 8 mice (at each time point) were sacrificed by cervical dislocation. Following the dissection of the tumors, the selection of the wavelength of the high performance liquid chromatography (HPLC) and the chromatographic condition, as well as the treatment of the sample were performed as previously described (19).

Indicators and methods for tumor detection. Fifty mice liver transplanted with cancer were randomly divided into 5 groups: the OCT-P407, OCT, ethanol, P407 and NS groups $(n=10)$, which were respectively injected with $\mathrm{P} 407$ plus $20 \mu \mathrm{g}$ of OCT, $20 \mu \mathrm{g}$ of OCT, $0.2 \mathrm{ml}$ of ethanol, P407 and $0.2 \mathrm{ml}$ of NS.

Detection of tumor volume. On day 0, 2, 4, 6 and 8 after injection, 3 orthogonal radial lines of the tumor, the length, width and thickness, were measured with a slide caliper rule; each radial line was measured 3 times and the values were averaged. Calculation of the tumor volume was carried out according to the formula: $\mathrm{V}=\pi / 6 \times \mathrm{a} \times \mathrm{b} \times \mathrm{c}(\mathrm{V}$, volume of the tumor; a, the longest radial line; $b$, the shortest radial line; $c$, thickness).

Detection of tumor weight and IR. All mice were culled 8 days after injection. Tumors were completely excised, weighed immediately and the tumor weight IR was calculated according to the formula: tumor weight IR $=(1-$ mean tumor weight of the drug administered group/mean tumor weight of the negative control group) $\mathrm{x} 100 \%$.

Mice from each group were sacrificed by cervical dislocation and the tumor tissue was isolated. After the tumors were weighed, part of them was immediately fixed in $10 \%$ neutral formalin solution and then embedded in paraffin. The other part of the tumor tissue was preserved in a liquid nitrogen container. Detection of the expression of somatostatin 
receptor-2 (SSTR-2), vascular endothelial growth factor (VEGF) and caspase-3 in the tumor tissue was performed by immunohistochemistry according to previous work in our laboratory (20).

Reverse transcription polymerase chain reaction $(R T-P C R)$. Extraction of total RNA and evaluation of RNA quality were carried out as described in our previous study (20). SSTR-2, VEGF, caspase- 3 and the control $\beta$-actin primers were designed by us in accordance with GenBank data: SSTR-2 (154 bp) forward, 5'-GAGGCCTTTCCCCTAGAGTT-3' and reverse, 5'-CACCGTAACGCTTGTCCTT-3'; VEGF (228 bp) forward, 5'-ATGGCAGGAGCCCCGGGGTGTCCC-3' and reverse, 5'-GGCTTGTCAATTTTTCTGGCTTTG-3'; caspase-3 (255 bp) forward, 5'-CAGACTCCGGCAGTAGTCGCC-3' and reverse, 5-GTGGACGCAGCCAACCTCAGA-3'; $\beta$-actin (266 bp) forward, 5'-ACAGAGTACTTGCGCTCAGGAG-3' and reverse, 5'-GTCACCCACACTGTGCCCATCT-3'. The one-step protocol was performed according to the RT-PCR (Thermo Fisher Scientific Inc.) manufacturer's instructions followed by semi-quantitative analysis.

Statistical analysis. Statistical software SPSS v17.0 was used for the analysis. Results are indicated as the means \pm SD . Measurements were performed in duplicate or triplicate and the t-test or one-way or two-way ANOVA analysis was applied. P-values $<0.05$ were considered to indicate statistically significant differences.

\section{Results}

\section{Cell culture experiments}

OCT-P407 effectively inhibits liver cancer cell proliferation. We first determined the optimum inhibitory concentration of OCT in OCT-P407 by testing the effects of various concentrations of OCT in OCT-P407 on liver cancer cell proliferation after $24 \mathrm{~h}$ of treatment. We observed that OCT-P407 containing OCT at a concentration range of $1-100 \mu \mathrm{g} / \mathrm{ml}$ inhibited the proliferation of Hca-F cells after $24 \mathrm{~h}$ in a concentration-dependent manner, with an IC50 of $20 \mu \mathrm{g} / \mathrm{ml}$ (Fig. 1A). OCT ( $20 \mu \mathrm{g} / \mathrm{ml})$ was therefore defined as the optimum inhibitory concentration for the OCT-P407 and OCT groups and used in all subsequent experiments. We then compared the IRs of OCT, OCT-P407 and $\mathrm{P} 407$ on liver cancer cell proliferation over time. The IR of OCT-P407 was significantly different $(\mathrm{P}<0.05)$ from the other groups and within the OCT-P407 group it was also significantly different $(\mathrm{P}<0.05)$ at $48 \mathrm{~h}$ when compared with 12 and $24 \mathrm{~h}$ of treatment (Fig. 1B).

OCT-P407 effectively induces liver cancer cell apoptosis. We compared the apoptotic rates of Hca-F cells in the absence of the solution and in the presence of OCT, OCT-P407 and P407 over time. The apoptotic rate of OCT-P407 was significantly different $(\mathrm{P}<0.05)$ compared with the other groups and within the OCT-P407 group it was also significantly different $(\mathrm{P}<0.05)$ at $48 \mathrm{~h}$ when compared with 12 and $24 \mathrm{~h}$ of treatment (Fig. 2).

OCT-P407 induces evident liver cancer cell morphological changes. We analyzed the morphological changes of Hca-F cells after $48 \mathrm{~h}$ of treatment with no solution, OCT, OCT-P407 and P407 under an inverted optical microscope. As was
A
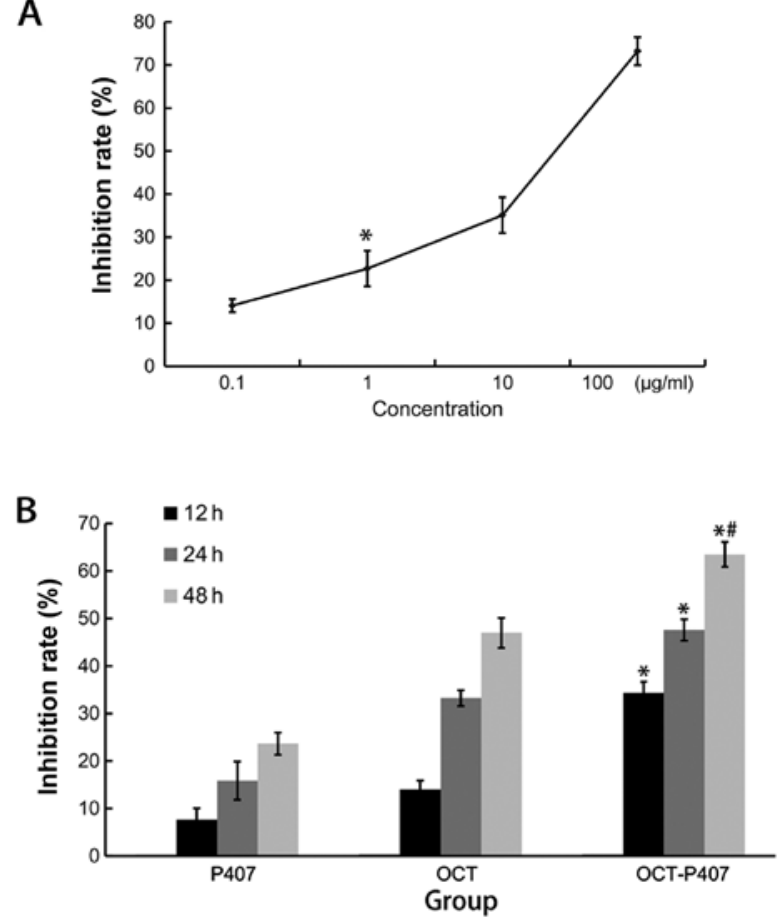

Figure 1. Inhibition of liver cancer cell proliferation upon treatment with octreotide (OCT)-poloxamer 407 (P407). (A) Hca-F cells were treated for $24 \mathrm{~h}$ with $\mathrm{P} 407$ and various concentrations of OCT and the inhibition rate (IR) was calculated, defining an IC50 for OCT of $20 \mu \mathrm{g} / \mathrm{ml}$. (B) Hca-F cells were treated for different periods of time with P407 or $20 \mu \mathrm{g} / \mathrm{ml}$ of OCT alone or in conjunction with P407 and the IR was calculated. Treatment with OCT-P407 inhibited cell proliferation more effectively that the other treatments $\left({ }^{*} \mathrm{P}<0.05\right)$, with the difference being significantly greater at $48 \mathrm{~h}$ compared with the previous time points $\left({ }^{\#} \mathrm{P}<0.05\right)$.

observed in the negative control group, the cells were roundshaped, with a transparent cytoplasm and they grew densely and were evenly distributed; in the P407 group, some cells formed clusters; while in the OCT-P407 group, they had an irregular shape, DNA condensation and fragmentation and the majority aggregated into clusters; in the OCT solution group, some of the cells showed morphological changes as mentioned above. Thus, in the OCT-P407 group, the morphological changes of the Hca-F cells were greater than those of cells in the OCT group and P407 group, as well as the negative control group $(\mathrm{P}<0.05)$. In the OCT group and $\mathrm{P} 407$ group the morphological changes of Hca-F cells were significantly greater than those in the negative control group $(\mathrm{P}<0.05)$. In Fig. 3, we present a bar graph illustrating the number of morphological changes observed in each group.

\section{Animal experiments}

P407 has a long retention time in the mouse tumor cavity. Methylene blue-P407 and methylene blue solution were injected into the tumor cavities of the mice that were subcutaneous transplanted with liver cancer cells. Our results revealed that mice that were injected with methylene blue-P407 had less effluent at the injection site and thereby a lighter blue-stained local skin, while mice that were injected with methylene blue solution had deeper blue-stained local skin due to a larger amount of effluent. Similar results were observed at 3 min after the injection, as well as $24 \mathrm{~h}$ later. When the solid tumor cavity 


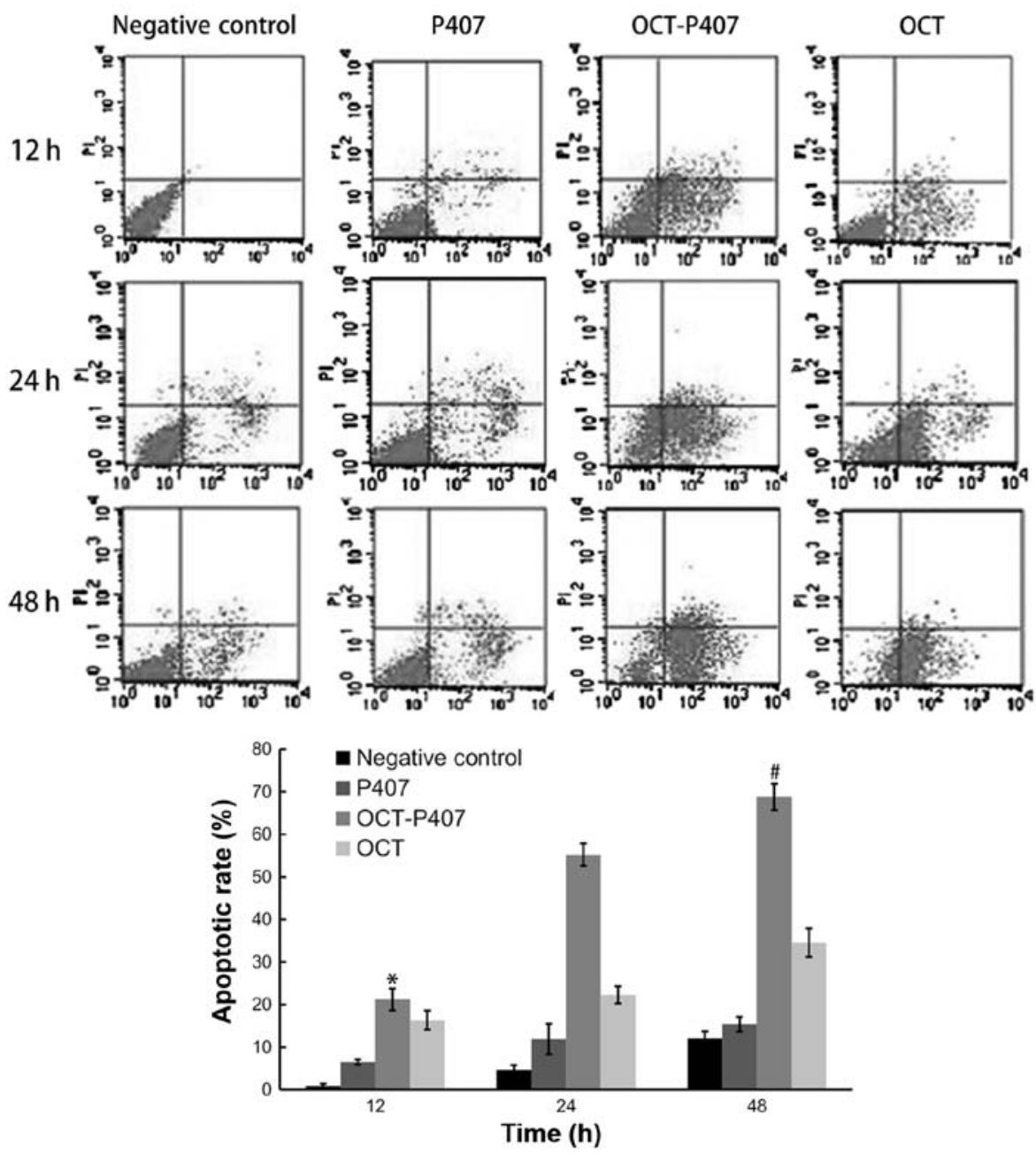

Figure 2. Induction of liver cancer cell apoptosis upon treatment with octreotide (OCT)-poloxamer 407 (P407). Hca-F cells were treated for different periods of time points with normal saline, P407 or $20 \mu \mathrm{g} / \mathrm{ml}$ of OCT alone or in conjunction with P407, stained with Annexin V-FITC/PI and analyzed by flow cytometry. The apoptotic rate of the different groups at the different time points was calculated as the percentage of Annexin V-FITC-positive/PI-negative cells. Treatment with OCT-P407 induced apoptosis more effectively than the other treatments ( $\mathrm{P}<0.05)$, with the difference being significantly greater at $48 \mathrm{~h}$ compared with the previous time points $\left({ }^{*} \mathrm{P}<0.05\right)$.

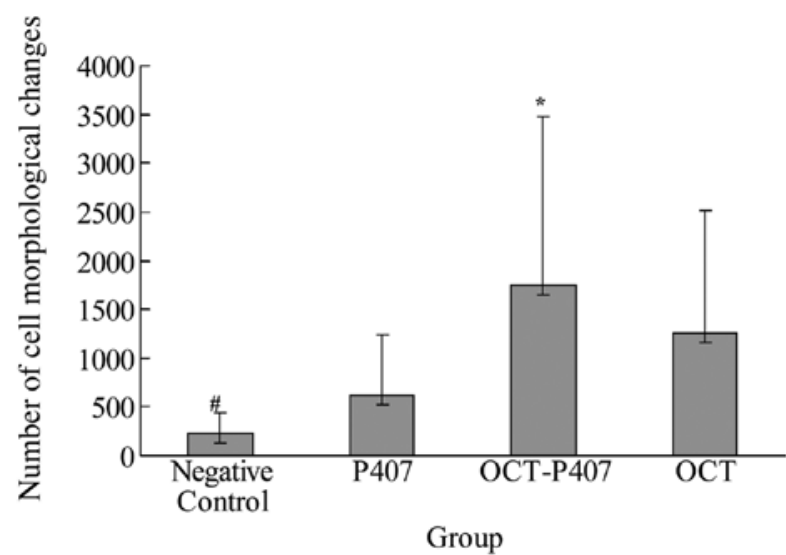

Figure 3. Bar graph showing the number of morphological changes of liver cancer cells upon treatment with OCT-P407 $(n=3)$. Hca-F cells were treated for $48 \mathrm{~h}$ with no solution, P407 or $20 \mu \mathrm{g} / \mathrm{ml}$ of OCT alone or in combination with P407 and cell morphological changes were observed under an inverted microscope. The number of morphological changes of Hca-F cells in the OCTP407 group was greater than that of cells in the OCT group and P407 group, as well as the negative control group $\left({ }^{*} \mathrm{P}<0.05\right)$. In the OCT group and $\mathrm{P} 407$ group, the number of morphological changes of Hca-F cells was significantly greater than that of cells in the negative control group $\left({ }^{\#} \mathrm{P}<0.05\right)$. was opened, we found a stronger pigmentation of methylene blue-P407 than methylene blue solution, indicating that the retention time of the former in the tumor cavity was longer than the latter (data not shown).

OCT-P407 has a large residual concentration in the mouse tumor cavity. At $12 \mathrm{~h}$ after the intratumoral injection of $100 \mu \mathrm{g} / \mathrm{ml}$ of OCT alone or in conjunction with P407, the peak areas (AU) of the 2 groups were detected by HPLC and the residual concentration of OCT (in $\mu \mathrm{g} / \mathrm{ml}$ ) was calculated by the external reference method. Our results revealed that the residual concentration of OCT in the OCT-P407 group was significantly higher than the OCT group $(\mathrm{P}<0.05)$. Moreover, at 24 and $48 \mathrm{~h}$ after injection, OCT could still be detected in the OCT-P407 group, but no was longer detected in the OCT solution group.

OCT-P407 prevents the increase in tumor volume and tumor weight. Before treatment there were no statistically significant differences in the tumor volume between the groups $(\mathrm{P}>0.05)$. As can be observed in Fig. 4B, the tumor volume in the untreated (NS) group increased gradually from day 0 to 8 after 
A

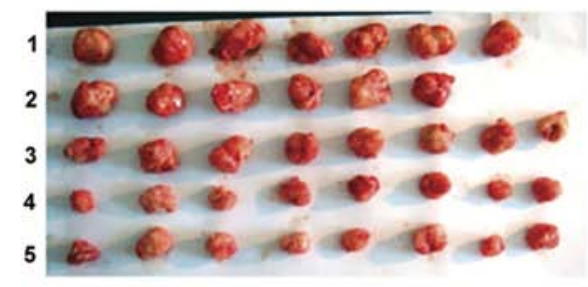

C

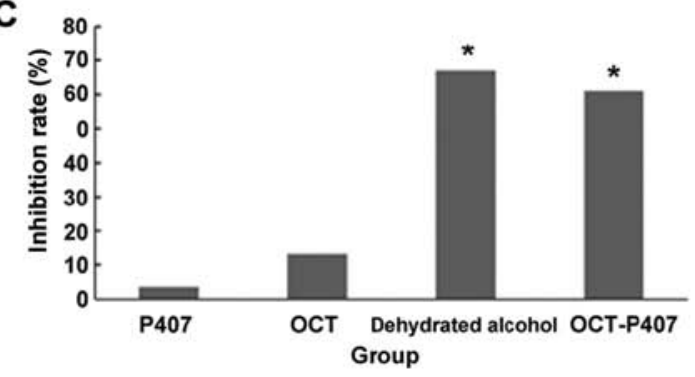

B

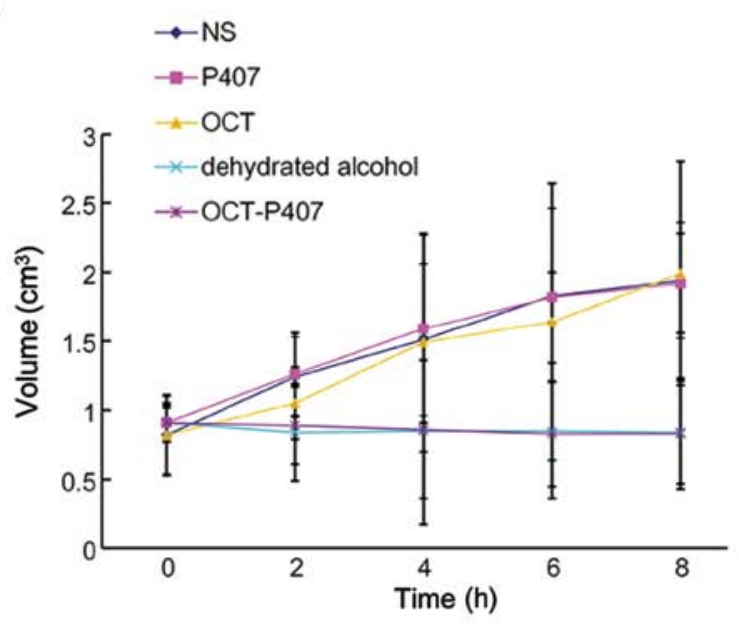

Figure 4. Changes in tumor volume and tumor weight upon treatment with octreotide (OCT)-poloxamer 407 (P407). (A) On day 8 of treatment with normal saline (NS), P407, OCT, ethanol and OCT-P407, the mice were culled and the tumors excised. Lane 1, NS group; lane 2, P407 group; lane 3, OCT group; lane 4, dehydrated alcohol group; lane 5, OCT-P407 group. (B) Tumor volume was measured at different time points, showing that the tumors in the OCT-P407 and ethanol groups were significantly smaller than those in the other groups $(\mathrm{P}<0.05)$. (C) On day 8 of treatment, tumor weight was measured and the tumor inhibition rate was calculated, showing that the tumors in the OCT-P407 and ethanol groups were significantly lighter than those in the other groups ( $\mathrm{P}<0.05)$.

administration, growing to twice the original size on day 8 . Changes in tumor volume in the P407 group were similar to those in the NS group, with these 2 groups presenting no significant differences $(\mathrm{P}<0.05)$ at all time points (Fig. 4B). On the second day after treatment, the tumor volume in the OCT group was not significantly altered compared with the volume before administration, but was significantly decreased when compared with the NS group $(\mathrm{P}<0.05)$. However, after 4,6 and 8 days of administration, tumor volume increased and there was no statistically significant difference compared with the NS group ( $\mathrm{P}>0.05$ ) (Fig. 4B). After 2, 4 and 6 days of administration, tumor volumes in the OCT-P407 group were not increased and on day 8 , there was no statistically significant difference compared with the volumes before administration (P>0.05) (Fig. 4B). Although on the second day after administration there was no significant difference between the tumor volumes in the OCT-P407 and OCT solution groups, from day 4 onwards, the tumor volume in the OCT group increased and the differences between these 2 groups became significant $(\mathrm{P}<0.05)$. Tumor volumes in the ethanol group did not increase over time and had no statistically significant differences when compared with the volumes before treatment $(\mathrm{P}>0.05)$ and also when compared with the tumor volumes in the OCT-P407 group at different time points ( $\mathrm{P}>0.05)$ (Fig. 4B).

After 8 days of treatment, the tumors were excised and we observed by the naked eye. The tumor volumes in the NS and P407 groups were larger, followed by the OCT solution group. The tumor volumes in the ethanol and OCT-407 groups were the lowest (Fig. 4A). Tumor weight was then measured and the tumor IR calculated. In accordance with the tumor volumes, tumors in the OCT-P407 and particularly those in the ethanol group were significantly lighter in weight $(\mathrm{P}<0.05)$ than those in the OCT, NS and P407 groups (Fig. 4C).

OCT-P407 induces protein expression of SSTR-2 and caspase-3 and decreases that of VEGF. As detected by immu- nohistochemistry of the mouse tumor sections, the number of positive cells, as well as the staining intensity of SSTR-2 and the number of cytoplasm yellow granules in the OCT-P407 group were significantly higher $(\mathrm{P}<0.05)$ than those in the OCT, P407 and NS groups and, even though there was no statistically significant difference $(\mathrm{P}>0.05)$, they were also higher than those in the ethanol group (Fig. 5). On the contrary, the number of positive cells, as well as the staining intensity of VEGF and the number of cytoplasm yellow granules in the OCT-P407 group were significantly lower $(\mathrm{P}<0.05)$ than those in the OCT, P407 and NS groups and, although there was no statistically significant difference $(\mathrm{P}>0.05)$, they were also lower than those of the ethanol group (Fig. 5). Similar to SSTR-2, the number of positive cells, as well as the staining intensity of caspase-3 and the number of cytoplasm yellow granules in the OCT-P407 group, were significantly higher $(\mathrm{P}<0.05)$ than those in the OCT, P407 and NS groups; however, they were lower, although not significantly ( $\mathrm{P}>0.05)$, than those in the ethanol group (Fig. 5).

OCT-P407 induces mRNA expression of SSTR-2 and caspase-3 and decreases that of VEGF. As detected by RT-PCR in the mouse tumor sections, the mRNA expression of SSTR-2 in the OCT-P407 group was significantly higher $(\mathrm{P}<0.05)$ than that in the OCT, P407, NS and even the ethanol group (Fig. 6). On the contrary, the mRNA expression of VEGF in the OCT-P407 group was significantly lower $(\mathrm{P}<0.05)$ than that in the OCT, P407 and NS groups and, but was not statistically significant compared with the ethanol group $(\mathrm{P}>0.05)$ (Fig. 6). Similar to SSTR-2, the mRNA expression of caspase-3 in the OCT-P407 group was significantly higher $(\mathrm{P}<0.05)$ than that in the OCT, P407, NS and even the ethanol group (Fig. 6).

\section{Discussion}

The incidence and mortality rates of primary hepatocellular carcinoma rank 7 th and 4th, respectively worldwide among 


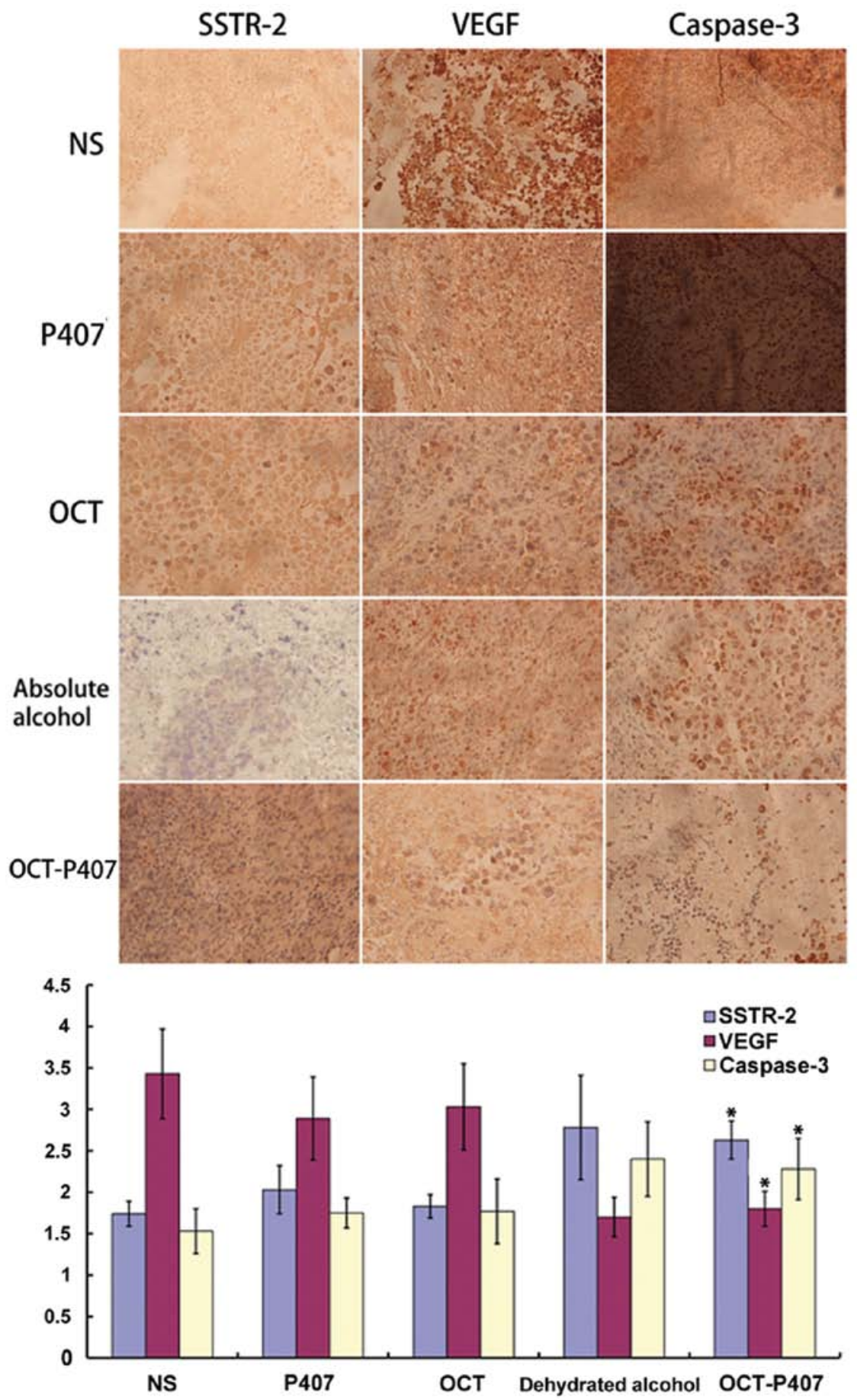

Figure 5. Protein levels of somatostatin receptor-2 (SSTR-2), vascular endothelial growth factor (VEGF) and caspase-3 upon treatment with octreotide (OCT)-poloxamer 407 (P407; magnification, x100). Protein levels of SSTR-2, VEGF and caspase-3 were detected by immunohistochemistry in tumor sections of mice treated with normal saline (NS), P407, OCT, ethanol and OCT-P407. OCT-P407-treated tumors displayed significantly higher protein levels of SSTR-2 and caspase-3 $\left({ }^{*} \mathrm{P}<0.05\right)$ and significantly lower protein levels of VEGF compared with the tumors treated with the other treatments $\left({ }^{*} \mathrm{P}<0.05\right)$.

tumors (21). Medication cannot improve the condition of the patients, whereas surgical procedures, such as hepatectomy or liver transplantation may be more efficient. However, even if the tumor is small and localized, the 5-year survival rate only reaches up to $37.9-50 \%(22,23)$. Studies have shown that the survival rate can be significantly improved when TACE is combined with physical or medical methodologies when treating solid tumors, such as PEI, liquid nitrogen freezing, high frequency laser, microwave and RFA among others. Progress has been made in TACE, positron emission tomography (PET) and RFA $(24,25)$ thus far, and studies have also begun to focus on percutaneous intratumoral injection chemotherapy (26-28). However, for those patients with advanced liver cancer with liver dysfunction and a poor physical condition, such treatments often have to be discontinued.

In recent years, OCT has been reported to have antitumor effects $(12,29-32)$. Compared with traditional chemotherapeutics, OCT has a better specificity, fewer side-effects and is harder to acquire resistance. Its action is mild and it has hepatoprotective effects (33). With these advantages, it is possible to 


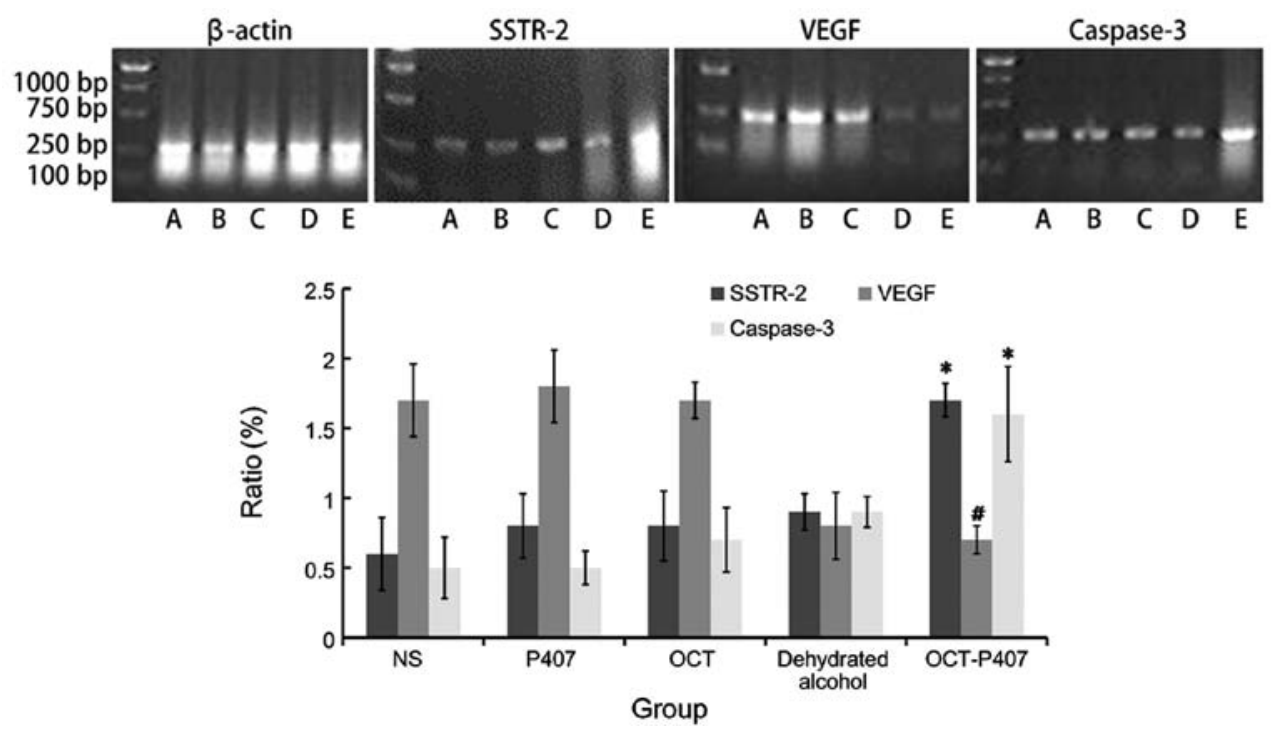

Figure 6. mRNA levels of somatostatin receptor-2 (SSTR-2), vascular endothelial growth factor (VEGF) and caspase-3 upon treatment with octreotide (OCT)-poloxamer 407 (P407). (A) Normal saline (NS); (B) P407; (C) OCT; (D) ethnaol; (E) OCT-P407. mRNA levels of SSTR-2, VEGF and caspase-3 were detected by RT-PCR in tumor sections of mice treated with NS, P407, OCT, ethanol and OCT-P407. OCT-P407-treated tumors display significantly higher $\left({ }^{*} \mathrm{P}<0.05\right)$ mRNA levels of SSTR-2 and caspase- 3 than all the other groups and significantly lower $\left({ }^{*} \mathrm{P}<0.05\right)$ mRNA levels of VEGF than those of the other groups, excluding the ethanol one.

conduct antitumor therapy in such patients mentioned above. In order to overcome the disadvantage of the short half-life of OCT, in the present study, we tried to prolong the action time of OCT and improve its treatment effectiveness by changing the pharmaceutical dosage form.

As a type of polymer, P407-based thermosensitive gels in situ have been extensively studied in recent years (34-36). It uses the difference in temperature between the environment and the human body to its advantage. It can be administered by injection and forms a gel at physiological temperatures, prolonging the action time of drugs, improving their therapeutic effects (28). In vivo and in vitro experiments have indicated that $\mathrm{P} 407$ has a stable release effect (37-41) and compared with other similar materials, it is more stable and can carry more drugs, in addition to the fact that its toxicity is low, its surface is smooth and soft and requires little stimulation. It will not be sensed as a foreign body on the injection site. Furthermore, it has a good biocompatibility and is easy to produce. It has been widely used in delayed and controlled release gel systems and it is an ideal and safe adjuvant used for the delayed release of drugs. P407 has been reported to be combined with adriacin, chitosan and other polymers to form depot preparations for intratumoral injection therapy (42-46). In the present study, considering that OCT has disadvantages, such as instability and a short half-life and that P407 may increase drug stability, we tried to combine these 2 materials to form the OCT-P407 depot preparation to enhance the effects of intratumoral injection for the treatment of hepatocellular carcinoma.

We conducted cell culture and in vivo experiments to investigate the antitumor effects of OCT-P407. First, MTT assay was used to detect the effects of OCT and OCT-P407 on the proliferation of Hca-F hepatocellular carcinoma cells. Our results indicated that the proliferation of liver cancer cells in the OCT solution group was inhibited. In our study, compared with the control group, $24 \mathrm{~h}$ after treatment of the Hca-F cells with OCT-P407 containing various concentrations of OCT, cell proliferation was markedly suppressed. With the increasing drug concentrations, the IR increased and once the best inhibitory concentration was defined, the inhibitory effects increased over time $(\mathrm{P}<0.05)$. The IRs of the OCT-P407 group upon 12, 24 and $48 \mathrm{~h}$ of treatment were significantly higher $(\mathrm{P}<0.05)$ than those of the OCT solution group. This may be due to the cumulative effects of drug delayed release that enhanced its effects.

Cell apoptosis is closely associated with the occurrence, development and malignant transformation of tumors. Studies have proven that OCT can not only inhibit the proliferation of tumor cells, but can also induce tumor cell apoptosis $(47,48)$. To assess this, we performed quantitative analysis of tumor cell apoptosis using the Annexin V-FITC/PI double staining method combined with flow cytometry. Our results indicated that the effects of OCT-P407 on the induction of apoptosis were significantly higher than those of OCT alone at different time points $(\mathrm{P}<0.05)$ and that these effects increased over time. We additionally observed the morphological changes in the liver cancer cells $48 \mathrm{~h}$ after the addition of OCT-P407 and OCT under an inverted light microscope and found that changes induced by OCT-P407 were more obvious.

Mouse livers are relatively small and therefore are not easy to operate on and observe and the Hca-F liver cancer cells used in this study grow better in lymphatic tissue. Moreover, establishing a mouse liver cancer implantation tumor model is easier, faster and reliable; therefore we used it as our in vivo experimental model.

It has been reported that the leaching method with no membrane is commonly used to investigate the release time of drugs in gels (49); however, this method is time consuming and can only be conducted in vitro. In this study, after establishing the mouse model of liver cancer by the subcutaneous 
transplantation of cancer cells, methylene blue and methylene blue thermosensitive gel were injected into the tumor cavity to investigate their retention at different time points. We observed a high amount of methylene blue in the methylene blue P407 group $24 \mathrm{~h}$ later compared with the methylene blue solution group. This experiment proved that P407 itself had an outstanding adhesive attraction that slowed down the flow speed of methylene blue in the solid tumor and prolonged its retention time in the solid tumor cavity, providing direct evidence for its delayed release effect in the tumor cavity. In this study, we injected prepared OCT-P407 or OCT solution into the tumor cavities of mice and observed the effects $12 \mathrm{~h}$ after injection. The OCT content in the mouse tumor cavities was higher in the OCT-P407 group than in the OCT solution group. In contrast to the OCT solution group, OCT could still be detected in the OCT-P407 group 24 and 48 h later. We concluded that the retention time of OCT-P407 was higher than that of OCT and this indicated that P407 has a delayed release effect.

Since our in vitro data have confirmed that OCT-P407 can inhibit liver cancer cell proliferation and induce tumor cell apoptosis, we proceeded to investigate its antitumor effects through in vivo experiments. We therefore compared OCT-P407 with ethanol, a well known therapeutic drug administered by intratumoral injection for the treatment of liver cancer, as well as with a conventional OCT preparation, NS and P407. These reagents were separately injected into mice subcutaneously transplanted with Hca-F hepatocellular carcinoma cells. As a result, the proliferation of tumor cells in the OCT-P407, ethanol and OCT groups was inhibited, with the tumor volumes at different time points after injection being smaller than those in the NS and $\mathrm{P} 407$ groups $(\mathrm{P}<0.05)$. Two days after the injection, tumor volumes in the OCT-P407 and ethanol groups were very similar to those in the OCT solution group $(\mathrm{P}<0.05)$; however, 4, 6 and 8 days after injection, their volumes were significantly lower than those in the OCT solution group $(\mathrm{P}<0.05)$. This indicated that the action time of OCT-P407 was significantly longer than that of OCT. Even though the tumor volume in the ethanol group tended to be lower than that in the OCT-P407 group, there was no statistically significant difference between them $(\mathrm{P}>0.05)$. In the end, the tumor weights in the OCT-P407 and ethanol groups were lower than those in the OCT, NS and P407 groups, although the tumor weight in the ethanol group was lower than that in the OCT-P407 group $(\mathrm{P}<0.05)$. Their IRs were 67 and $61 \%$, respectively, with the difference between them being statistically significant $(\mathrm{P}<0.05)$. Our results confirmed that OCT-P407 had a greater inhibitory effect on tumor cell proliferation than OCT and that its effects were similar to those of ethanol as regards tumor volume, although its effects were not as prominent as those of the latter as regards tumor weight and IR. This may be due to the central tumor necrosis caused by ethanol.

Previous studies have suggested that the antitumor mechanism of OCT may be related to the following factors: i) The direct inhibition of tumor cell proliferation, which is related to the expression of the SSTR on the tumor cell surface (50). In 1998, Kouroumalis et al (51) found that there were SSTRs in human liver cancer cells contrary to normal liver tissue. The combination of OCT and SSTR produces vasoconstrictive effects and induces a dysfunction of the blood circulation in tumor tissue. It has been described that there are one or more types of SSTRs in the majority of tumor cells, which can be inhibited by OCT and SSTR-2 is the most common. OCT has a great affinity for SSTR-2 and it is its selective receptor antagonist. The combination of OCT and SSTR can exert cytotoxic effects, thereby inducing a lethal effect on target cells $(51,52)$. ii) The inhibition of angiogenesis, which inhibits tumor cell proliferation by reducing tumor blood supply. It has been reported that OCT can also reduce angiogenesis by inhibiting VEGF (53). VEGF is the strongest vascular growth factor with the highest specificity. It acts on vascular endothelial cells specifically, participates in the angiogenesis of solid tumors and it is closely associated with the biological characteristics of solid tumors, such as invasion and metastasis $(54,55)$. iii) The induction of tumor cell apoptosis. It has been described that one of the antitumor mechanisms of OCT may be the induction of the apoptosis of malignant cells $(56,57)$. The core component of the apoptotic cascade is the cysteinyl aspartate specific proteinase (caspase) family (58), with caspase-3 being the crucial effector molecule in this process $(59,60)$.

In this study, we investigated the mRNA and protein expression of SSTR-2, VEGF and caspase-3 and found that, compared with the ethanol group, the OCT-P407 group tended to have increased mRNA and protein expression levels of SSTR-2 and caspase-3; however, there was no statistically significant difference $(\mathrm{P}>0.05)$. The difference was however, significant when compared with the OCT solution, P407 and NS groups $(\mathrm{P}<0.05)$. The expression of VEGF protein in the OCT-P407 group tended to be lower than that in the ethanol group, despite the absence of significance $(\mathrm{P}>0.05)$. VEGF mRNA and protein expression in the OCT-P407 group was lower than that in the OCT solution, P407 and NS groups $(\mathrm{P}<0.05)$, but higher than that in the ethanol group as regards mRNA expression $(\mathrm{P}<0.05)$.

Taken together, our results indicate that the delayed release effect of OCT-P407 leads to a stronger antitumor effect than OCT alone and that the mechanisms involved may be: i) Upregulation of the expression of SSTR-2, inhibiting the growth of liver cancer cells and inducing apoptosis. It has been shown that with the application of an osmotic micropump containing OCT, the long-term sustaining release of OCT can lead to the upregulation of SSTR-2 and in tumor models, this upregulation of SSTR-2 depends on the continuous contact with OCT, demonstrating that the sustained release of small doses of OCT can lead to the upregulation of SSTR-2 in tumor cells (61). ii) OCT-P407 can enhance the inhibitory effects on angiogenesis by enhancing the inhibitory effects of OCT on the gene and protein expression of VEGF. The occurrence and development of liver cancer are closely related to the expression levels of VEGF $(57,62)$. The delayed release effect of OCT-P407 can inhibit the occurrence and development hepatocellular carcinoma. iii) OCT-P407 can induce apoptosis through the signal transduction pathway mediated by caspase-3. OCT-P407 is similar to ethanol in its antitumor mechanism. The only difference between them was that the mRNA expression level of VEGF was lower in the ethanol group than the OCT-P407 group, indicating that ethanol may inhibit angiogenesis faster and more prominently by causing tissue necrosis.

As stated above, in the present study, firstly, we proved by in vitro cell culture experiments that OCT-P407 inhibits the 
proliferation and induces the apoptosis of liver cancer cells. We then showed that the retention time of OCT-P407 in the solid tumor cavity is longer than that of OCT, which indicates that OCT-P407 has a delayed release effect. We also observed that even though the antitumor effects of the intratumoral injection of OCT-P407 were not as effective as those of ethanol in general, in some aspects they had similar results. This suggests that OCT-P407 may prove to be effective as an antitumor drug. There were some drawbacks in this experiment, such as the ratio of the concentration of the thermosensitive gel and cell culture medium which was difficult to handle in the in vitro cell culture experiments and thus 3 days after the gels were added, the medium became muddy and difficult to observe. Also, in the in vivo experiments, the livers of the mice were too small to obtain tumor tissue transplants and we were thus unable to establish the liver solid tumor model. Moreover, in the design of the experiment, a chemotherapeutics group was meant to be included in the control groups; however, due to the variety of drugs already described, we decided not to include such a group. To date, only a few cell culture experiments using gels have been performed by us and others and we hope that the experimental methodology can be improved in the future. Our laboratory will select a more appropriate model of liver cancer solid tumors for further experiments and we are looking forward to providing patients with a new advanced liver cancer treatment with the advantages of delayed release, low toxicity and better compliance.

\section{Acknowledgements}

The present study was supported by grants from the National Natural Science Foundation of China (no. 30970886) and the Science and Technology Project of Dalian (no. 2010E15SF179).

\section{References}

1. Han KH and Park JY: Systemic treatment in advanced/metastatic hepatocellular carcinoma in the era of targeted therapy. J Gastroenterol Hepatol 25: 1023-1025, 2010.

2. Zhang ZM, Guo JX, Zhang ZC, Jiang N, Zhang ZY and Pan LJ: Therapeutic options for intermediate-advanced hepatocellular carcinoma. World J Gastroenterol 17: 1685-1689, 2011.

3. Bouza C, López-Cuadrado T, Alcázar R, Saz-Parkinson Z and Amate JM: Meta-analysis of percutaneous radiofrequency ablation versus ethanol injection in hepatocellular carcinoma. BMC Gastroenterol 9: 31, 2009.

4. Burton KR, O'Dwyer H and Scudamore C: Percutaneous ethanol ablation of hepatocellular carcinoma: periprocedural onset alcohol toxicity and pancreatitis following conventional percutaneous ethanol ablation treatment. Can J Gastroenterol 23 554-556, 2009.

5. Danila M, Sporea I, Sirli R and Popescu A: Percutaneous ethanol injection therapy in the treatment of hepatocarcinoma-results obtained from a series of 88 cases. J Gastrointestin Liver Dis 18: 317-322, 2009.

6. Weijian F, Zan L, Suhong H, et al: Destructive effect of percutaneous hydrochloric acid injection therapy for liver cancer - a preliminary experimental and clinical study. Gan To Kagaku Ryoho 33: 1852-1856, 2006.

7. Castro DJ, Sridhar KS, Garewal HS, et al: Intratumoral cisplatin/ epinephrine gel in advanced head and neck cancer: a multicenter randomized, double-blind, phase III study in North America. Head Neck 25: 717-731, 2003.

8. Kwak MK, Hur K, Yu JE, et al: Suppression of in vivo tumor growth by using a biodegradable thermosensitive hydrogel polymer containing chemotherapeutic agent. Invest New Drugs 28: 284-290, 2010.
9. Bornschein J, Drozdov I and Malfertheiner P: Octreotide LAR: safety and tolerability issues. Expert Opin Drug Saf 8: 755-768, 2009.

10. Guo TK, Hao XY, Ma B, et al: Octreotide for advanced hepatocellular carcinoma: a meta-analysis of randomized controlled trials. J Cancer Res Clin Oncol 135: 1685-1692, 2009.

11. Zhang J, Jin W, Wang X, Wang J, Zhang X and Zhang Q: A novel octreotide modified lipid vesicle improved the anticancer efficacy of doxorubicin in somatostatin receptor 2 positive tumor models. Mol Pharm 7: 1159-1168, 2010.

12. Wu LQ, Lu Y,Lu HJ,Zhao ZG and Yang M: Efficacy of intra-tumor injection of Kang-Lai-Te in treating transplanted hepatoma in rats. Hepatobiliary Pancreat Dis Int 3: 580-584, 2004.

13. Liu S, Sun MZ, Tang JW, Wang Z, Sun C and Greenaway FT: High-performance liquid chromatography/nano-electrospray ionization tandem mass spectrometry, two-dimensional difference in-gel electrophoresis and gene microarray identification of lymphatic metastasis-associated biomarkers. Rapid Commun Mass Spectrom 22: 3172-3178, 2008.

14. Yu SJ, Kang XH, Zhang JN, et al: Effects of small interfering RNA targeting heparanase-1 combined with heparin on invasiveness of mouse hepatocellular carcinoma cell lines. Chin J Cancer 29: 816-823, 2010.

15. Sasaki K, Tsuno NH, Sunami E, et al: Chloroquine potentiates the anti-cancer effect of 5-fluorouracil on colon cancer cells. BMC Cancer 10: 370, 2010.

16. Aresvik DM, Pettersen RD, Abrahamsen TG and Wright MS: 5-fluorouracil-induced death of Jurkat T-cells-a role for caspases and MCL-1. Anticancer Res 30: 3879-3887, 2010.

17. Song B, Tang JW, Wang B, Cui XN, Zhou CH and Hou L: Screening for lymphatic metastasis-associated genes in mouse hepatocarcinoma cell lines Hca-F and Hca-P using gene chip. Ai Zheng 24: 774-780, 2005 (In Chinese).

18. Cui XN, Tang JW, Song B, Wang B, Chen SY and Hou L: High expression of osteoglycin decreases gelatinase activity of murine hepatocarcinoma Hca-F cells. World J Gastroenterol 15: 6117-6122, 2009.

19. Ballal N, Kundabala M, Bhat K, et al: Susceptibility of Candida albicans and Enterococcus faecalis to Chitosan, Chlorhexidine gluconate and their combination in vitro. Aust Endod J 35: 29-33, 2009.

20. Guo SB, Duan ZJ, Li Q and Sun XY: Effect of heme oxygenase-1 on renal function in rats with liver cirrhosis. World $\mathrm{J}$ Gastroenterol 17: 322-328, 2011.

21. International Agency for Research on Cancer: World Hearth Organization: WHO IARC Report.

22. Alkofer B, Lepennec V and Chiche L: Hepatocellular cancer in the non-cirrhotic liver. J Visc Surg 148: 3-11, 2011.

23. Choi GH, Park JY, Hwang HK, et al: Predictive factors for long-term survival in patients with clinically significant portal hypertension following resection of hepatocellular carcinoma. Liver Int 31: 485-493, 2011.

24. Johnson PJ: Non-surgical treatment of hepatocellular carcinoma. HPB (Oxford) 7: 50-55, 2005.

25. Kurokohchi K, Hosomi N, Yoshitake A, et al: Successful treatment of large-size advanced hepatocellular carcinoma by transarterial chemoembolization followed by the combination therapy of percutaneous ethanol-lipiodol injection and radiofrequency ablation. Oncol Rep 16: 1067-1070, 2006.

26. Jang JW, Park YM, Bae SH, et al: Therapeutic efficacy of multimodal combination therapy using transcatheter arterial infusion of epirubicin and cisplatin, systemic infusion of 5-fluorouracil, and additional percutaneous ethanol injection for unresectable hepatocellular carcinoma. Cancer Chemother Pharmacol 54: 415-420, 2004.

27. Oh YJ, Park YM, Kim BH, et al: A case of hepatocellular carcinoma with pulmonary metastases treated successfully with a combination of repeated hepatic arterial infusion epirubicin and Cisplatin chemotherapy and systemic low-dose infusion of 5-Fluorouracil. Gut Liver 3: 343-348, 2009.

28. Yang L, Wang B, Qiao W and Liu P: A novel combination chemotherapy integrating with intratumoral chemotherapy. Medical hypotheses 73: 334-335, 2009.

29. Chen S, Xie Y, Wang CH and Tang CW: [Effects of octreotide on necrosis of hepatocellular carcinoma xenografts in nude mice]. Ai Zheng 28: 673-678, 2009 (In Chinese).

30. Varas-Lorenzo MJ: Long-standing malignant pancreatic carcinoid treated with octreotide. Rev Esp Enferm Dig 102: 662-666, 2010. 
31. Pettit L and El-Modir A: The role of somatostatin analogues in the treatment of advanced malignant thymomas: case report and review of the literature. Br J Radiol 84: e7-e10, 2011.

32. Takeuchi K, Fujiwara K, Tsujino T and Morita H: Successful medical treatment with octreotide for chyloperitoneum following paraaortic lymphadenectomy in the treatment of gynecologic malignancies: a report of 2 cases. J Reprod Med 56: 75-77, 2011

33. Tracy TF Jr, Tector AJ, Goerke ME, Kitchen S and Lagunoff D Somatostatin analogue (octreotide) inhibits bile duct epithelial cell proliferation and fibrosis after extrahepatic biliary obstruction. Am J Pathol 143: 1574-1578, 1993.

34. Cao Y,Zhang C, Shen W, Cheng Z, Yu LL and Ping Q: Poly(N-isop ropylacrylamide)-chitosan as thermosensitive in situ gel-forming system for ocular drug delivery. J Control Release 120: 186-194, 2007.

35. Batrakova EV and Kabanov AV: Pluronic block copolymers: evolution of drug delivery concept from inert nanocarriers to biological response modifiers. J Control Release 130: 98-106, 2008.

36. Liu Y, Zhu YY, Wei G and Lu WY: Effect of carrageenan on poloxamer-based in situ gel for vaginal use: improved in vitro and in vivo sustained-release properties. Eur J Pharm Sci 37: 306-312, 2009.

37. Chen Y, Li L, Liu H, Peng B and Jin R: Preparation and release in vitro of injectable thermosensitive in situ gel of Glabrous Sarcandra herb extract. Zhongguo Zhong Yao Za Zhi 34: 2586-2589, 2009 (In Chinese).

38. Ammar HO, Salama HA, Ghorab $M$ and Mahmoud AA: Development of dorzolamide hydrochloride in situ gel nanoemulsion for ocular delivery. Drug Dev Ind Pharm 36: 1330-1339, 2010.

39. Baloğlu E, Karavana SY, Hyusein IY and Köse T: Design and formulation of mebeverine $\mathrm{HCl}$ semisolid formulations for intraorally administration. AAPS PharmSciTech 11: 181-188, 2010.

40. Monti D, Burgalassi S, Rossato MS, et al: Poloxamer 407 microspheres for orotransmucosal drug delivery. Part II: In vitro/ in vivo evaluation. Int J Pharm 400: 32-36, 2010.

41. Kojarunchitt T, Hook S, Rizwan S, Rades T and Baldursdottir S: Development and characterisation of modified poloxamer 407 thermoresponsive depot systems containing cubosomes. Int $\mathrm{J}$ Pharm 408: 20-26, 2011.

42. Ishihara M, Obara K, Nakamura S, et al: Chitosan hydrogel as a drug delivery carrier to control angiogenesis. J Artif Organs 9: 8-16, 2006

43. Guo DD, Moon HS, Arote R, et al: Enhanced anticancer effect of conjugated linoleic acid by conjugation with Pluronic F127 on MCF-7 breast cancer cells. Cancer Lett 254: 244-254, 2007.

44. Sastre RL, Olmo R, Teijón C, Muñíz E, Teijón JM and Blanco MD: 5-Fluorouracil plasma levels and biodegradation of subcutaneously injected drug-loaded microspheres prepared by spray-drying poly(D,L-lactide) and poly(D,L-lactide-co-glycolide) polymers. Int J Pharm 338: 180-190, 2007.

45. Al-Abd AM, Hong KY, Song SC and Kuh HJ: Pharmacokinetics of doxorubicin after intratumoral injection using a thermosensitive hydrogel in tumor-bearing mice. J Control Release 142: 101-107, 2010

46. Bae WK, Lee JH, Lee SJ, et al: Enhanced anti-cancer effect of 5-fluorouracil loaded into thermo-responsive conjugated linoleic acid-incorporated poloxamer hydrogel on metastatic colon cancer models. J Nanosci Nanotechnol 11: 1425-1428, 2011.
47. Liu HL, Huo L and Wang L: Octreotide inhibits proliferation and induces apoptosis of hepatocellular carcinoma cells. Acta Pharmacol Sin 25: 1380-1386, 2004.

48. Hua YP, Yin XY, Peng BG, et al: Mechanisms and influence of octreotide-induced regulation of somatostatin receptor 2 on hepatocellular carcinoma. Chemotherapy 55: 312-320, 2009.

49. Moore T, Croy S, Mallapragada S and Pandit N: Experimental investigation and mathematical modeling of Pluronic F127 gel dissolution: drug release in stirred systems. J Control Release 67: 191-202, 2000.

50. Susini C and Buscail L: Rationale for the use of somatostatin analogs as antitumor agents. Ann Oncol 17: 1733-1742, 2006.

51. Kouroumalis E, Skordilis P, Thermos K, Vasilaki A, Moschandrea $\mathbf{J}$ and Manousos ON: Treatment of hepatocellular carcinoma with octreotide: a randomised controlled study. Gut 42: 442-447, 1998.

52. Patel YC: Somatostatin and its receptor family. Front Neuroendocrinol 20: 157-198, 1999.

53. Jia WD, Xu GL, Xu RN, et al: Octreotide acts as an antitumor angiogenesis compound and suppresses tumor growth in nude mice bearing human hepatocellular carcinoma xenografts. J Cancer Res Clin Oncol 129: 327-334, 2003.

54. Zhang ZL,Liu ZS and Sun Q: Expression of angiopoietins, Tie2 and vascular endothelial growth factor in angiogenesis and progression of hepatocellular carcinoma. World J Gastroenterol 12: 4241-4245, 2006

55. Kandalaft LE, Motz GT, Busch J and Coukos G: Angiogenesis and the tumor vasculature as antitumor immune modulators: the role of vascular endothelial growth factor and endothelin. Curr Top Microbiol Immunol 344: 129-148, 2011.

56. Ferrante E, Pellegrini C, Bondioni S, et al: Octreotide promotes apoptosis in human somatotroph tumor cells by activating somatostatin receptor type 2. Endocr Relat Cancer 13: 955-962, 2006.

57. Kaseb AO, Hanbali A, Cotant M, Hassan MM, Wollner I and Philip PA: Vascular endothelial growth factor in the management of hepatocellular carcinoma: a review of literature. Cancer 115: 4895-4906, 2009.

58. McNeish IA, Bell S, McKay T, Tenev T, Marani M and Lemoine NR: Expression of Smac/DIABLO in ovarian carcinoma cells induces apoptosis via a caspase-9-mediated pathway. Exp Cell Res 286: 186-198, 2003.

59. Krajewska M, Wang HG,KrajewskiS, et al: Immunohistochemical analysis of in vivo patterns of expression of CPP32 (Caspase-3), a cell death protease. Cancer Res 57: 1605-1613, 1997.

60. Tsagarakis NJ, Drygiannakis I, Batistakis AG, Kolios G and Kouroumalis EA: Octreotide induces caspase activation and apoptosis in human hepatoma HepG2 cells. World J Gastroenterol 17: 313-321, 2011.

61. Plate KH, Breier G, Weich HA, Mennel HD and Risau W: Vascular endothelial growth factor and glioma angiogenesis: coordinate induction of VEGF receptors, distribution of VEGF protein and possible in vivo regulatory mechanisms. Int $\mathrm{J}$ Cancer 59: 520-529, 1994.

62. Tammela T, Zarkada G, Wallgard E, et al: Blocking VEGFR-3 suppresses angiogenic sprouting and vascular network formation. Nature 454: 656-660, 2008. 